Proc. Indian Acad. Sci. (Earth Planet. Sci.), Vol. 103, No. 1, March 1994, pp. 1-15. (C) Printed in India.

\title{
A critical analysis of processes governing the nutrient profiles in the ocean
}

\author{
R RAMESH* and D LAL** \\ * Physical Research Laboratory, Ahmedabad 380009, India \\ * Scripps Institution of Oceanography, La Jolla, CA 92093-0220, USA
}

MS received 13 September 1993; revised 6 January 1994

\begin{abstract}
A betract. We have theoretically considered the problem of interpretation of nutrient profiles in the upper ocean $(100-2000 \mathrm{~m})$. We compare the experimental depth profiles of nonconservative tracers, both stable and radioactive, with solutions of one-dimensional steady state transport equations of increasing complexity including situations not encountered in the real oceans. A part from gaining insight into the nutrient transport processes, this analysis is useful in offering a way to obtain operational estimates of depth dependent/independent eddy diffusivity and dissolution huxes in the ocean. These parameters are essential for estimating new production, total production and burial of carbon in the sediments.
\end{abstract}

Keywords Nutrients; conservative tracers; non-conservative tracers; eddy diffusion; advection; radiotracers; stable tracers; tracer transport; new production.

\section{Introduction}

One of the central problems in biological and chemical oceanography is the circulation of nutrients and trace elements in the oceans, and their relation to new production, total production and burial of carbon in the sediments. In the past, several tracers have been used to understand these processes and for obtaining their time scales. In this presentation, we specifically discuss the information which can be obtained on processes in the upper ocean $(100-2000 \mathrm{~m})$, using a suite of tracers; conservative as well as non-conservative, stable and radioactive.

Although tracers have been used for a long time (Broecker and Peng 1982), the first informative discussion of the theoretical basis for the use of conservative tracers was presented by Munk (1966). He showed using a vertical (one dimension) model that conservative stable tracers, e.g., temperature and salinity, can provide a useful estimate of the quantity $W / K(W$, vertical advection velocity, $K$ addy diffusivity), and that a conservative radiotracer (e.g., ${ }^{14} \mathrm{C}$ ) provides an estimate of $W^{2} / K$. Thus from a simultaneous use of two tracers, one can separately estimate $W$ and $K$, if indeed the use of a one-dimensional vertical (1-D) model can be justified.

It is often convenient and even more meaningful in the case of some tracers, to use a 1-D model eventhough one realizes its limitations. The distribution of a tracer, depending on its source function and physical and chemical behaviour, is often governed by mixing/transport processes in one or the other spatial domains. For example, ${ }^{7} \mathrm{Be}$ (half life $=53.3$ days) is introduced uniformly over the ocean surface. Its concentrations are measurable only in the near surface waters, at depths $<200 \mathrm{~m}$ (Silker 1972; Lee et al 1992) and its distribution in oceans is primarily determined 
by the vertical eddy diffusivity. Although the element beryllium participates in the nutrient cycles, it can be shown that the vertical distribution of ${ }^{7} \mathrm{Be}$, modeling its vertical transport by biological processes, based on rate constants determined from ${ }^{10} \mathrm{Be}$ profiles in sea water, is primarily controlled by eddy diffusion. In a recent paper (Ramesh and Lal, in preparation), the principal pathways for mixing and transport of different tracers are discussed. It is shown that from the radiotracers ${ }^{32} \mathrm{P},{ }^{33} \mathrm{P},{ }^{7} \mathrm{Be}$ and ${ }^{32} \mathrm{Si}$, vertical 1-D model suffices to characterize their profiles. The same holds for the nutrients $P, N$ and $\mathrm{Si}$. However, both the nutrient $\mathrm{C}$ and radiotracer ${ }^{14} \mathrm{C}$, 3-D models are needed to fully understand their distribution in the different oceans.

It should also be pointed out that tracer data are not usually extensive and/or accurate enough to employ 3-D models. Secondly, the 3-D models introduce additional unknowns, e.g., diffusivity in three dimensions. Considering these factors we believe that one can obtain sufficient useful operational estimates of the magnitude and variations of relevant mixing and biological parameters, whose validity can often be checked using radiotracers of different chemical behavior and half lives. In this paper we adopt the 1-D model and extend the discussions of Munk (1966) to non-conservative stable and radiotracers, with a view to quantify biological production and nutrient recycling in the upper layers of the oceans. We consider this to be a useful exercise because most of the available tracers are non-conservative in nature, except for the isotopes of hydrogen and oxygen $\left(\mathrm{D},{ }^{3} \mathrm{H},{ }^{18} \mathrm{O}\right)$. But these tracers do not show appreciable depth variations in the oceans because of small differences between the 'source' and 'sink' regions. Their evolution is governed by equilibrium between diffusive and advective mixing operative over large space scales. The usefulness of these isotopes lies in providing information on water masses originating from different regions in the ocean. For information on processes in the upper ocean, one has to resort to radiotracers, conservative and/or non-conservative. Unfortunately, the conservative radiotracer, ${ }^{3} \mathrm{H}$, is not applicable for studying nutrient cycles and biological production.

We show that using one-dimensional models should be possible in certain cases to estimate the physical and chemical parameters related to nutrient dynamics (by a suitable combination of different tracers). The limitations of the present approach are obvious; nevertheless, no other comparable methods are presently available.

\section{Tracer models}

Below we consider the tracer transport/mixing and mass balance equations for conservative and non-conservative tracers. We deal with steady state situations; this implies that the net inward flux into the mixing zone under consideration is balanced by the net outward flux from the zone (for non-conservative stable tracers); and also by the radioactive decay (for non-conservative radiotracers). This would also imply that the euphotic zone above the mixing zone does not receive any nutrients from or lose nutrients to the adjacent ocean water masses by horizontal mixing. We adopt the following nomenclature:

$z \quad$ Depth below the mixed layer (positive downwards).

$C$ Concentration of a stable tracer (at depth $z$ ).

$C^{*}$ Concentration of a radiotracer (at depth $z$ ). (Subscripts 1 and 2 denote the values at the top and bottom of the depth range considered). 
$K \quad$ Vertical eddy diffusivity $\left(K_{0}\right.$ is the constant value applicable for deep ocean and $K_{W}$ is the wind driven depth dependent component at zero depth).

$W \quad$ Vertical advection velocity.

$J$ Dissolution flux (time rate of dissolution per unit volume of water) of a stable tracer (at depth $z$ ).

$J^{*} \quad$ Dissolution flux of a radiotracer (at depth $z$ ).

(again, subscript 0 denotes the value at zero depth).

$\lambda$ Disintegration constant for a radiotracer.

$T$ Temperature.

$S \quad$ Salinity.

The parameters $a, b, c, \ldots h$, and $p, q, r, s$ are arbitrary constants determined using boundary conditions.

\subsection{Conservative stable tracers (CS), e.g., T, S (1-D model)}

Following Munk (1966), the steady state 1-D equation:

$$
K_{0} \frac{\partial^{2} T}{\partial z^{2}}-W_{0} \frac{\partial T}{\partial z}=0
$$

has the solution:

$$
T(z)=a\left[\exp \left(\frac{W_{0}}{K_{0}} z\right)\right]-b \frac{W_{0}}{K_{0}}
$$

where the constants $a$ and $b$ are determined from boundary conditions. In equation (1), $K$ and $W$ are assumed to be depth invariant (i.e., $K=K_{0}$ and $J=J_{0}$ ). In this case the tracer depth profile yields the ratio $W_{0} / K_{0}$ within a given water mass. The ratio $K_{0} / W_{0}$ has the dimension of length, and specifies the mean distance for balance in the tracer concentration which is maintained by the opposing influences of eddy diffusivity and advection. No direct information can however be expected from a stable conservative tracer about the rate constants of any of the processes governing equilibrium in the system.

\subsection{Conservative radiotracers $(C R)$}

For a conservative radiotracer, the steady state 1-D equation (Munk 1966):

$$
K_{0} \frac{\partial^{2} C}{\partial z^{2}}-W_{0} \frac{\partial C}{\partial z}-\lambda C=0
$$

has the solution:

where

$$
C(z)=a[\exp (\alpha z)]+b[\exp (\beta z)]
$$

$$
\alpha, \beta=\frac{W_{0}}{2 K_{0}}\left[1 \pm\left\{1+\frac{4 \lambda K_{0}}{W_{0}^{2}}\right\}^{1 / 2}\right]
$$

which contains two different ratios with the parameters $W_{0}$ and $K_{0}: W_{0} / K_{0}$ and $K_{0} / W_{0}^{2}$. Munk (1966) suggested that since $W_{0} / K_{0}$ can be obtained using a stable 
conservative tracer, the conservative radiotracer data could be used to yield the quantity $K_{0} / W_{0}^{2}$ and hence $K_{0}$ and $W_{0}$ can be estimated separately.

Note, however, that generally no suitable radiotracers are available to achieve the goal. For example the commonly employed long-lived radiotracers, ${ }^{14} \mathrm{C},{ }^{226} \mathrm{Ra}$ and ${ }^{32} \mathrm{Si}$ are all non-conservative (Lal 1962; Craig 1969; Chung and Craig 1973; Somayajulu et al 1987). Use of these non-conservative tracers, however, introduces the additional term $J$ in equation (3), signifying their dissolved flux at depths due to dissolution of sinking organic particulate matter. Munk (1966) did not consider the impact of $J$ term in his classic treatment of tracer equations. Also, he did not consider the depth variations in eddy diffusivity, which must be incorporated in any tracer equation, especially in the upper regions of the ocean. In the following we will consider non-conservative tracer models with depth dependent $K$ and $J$. Reference is made here to Craig (1969) who presented detailed solutions of 1-D transport model for non-conservative radiotracers in the ocean.

\subsection{Non-conservative stable (NCS) and non-conservative radioactive (NCR) tracers}

The steady state equation for a non-conservative tracer (NCR) is:

$$
\frac{\partial}{\partial z}\left\{K \frac{\partial C}{\partial z}\right\}-W \frac{\partial C}{\partial z}-\lambda C+J=0
$$

By setting $\lambda$ and/or $J=0$, one obtains the corresponding equations for conservative stable (CS), non-conservative stable (NCS) and conservative radiotracers (CR).

We will now consider the case of non-conservative tracers in the order of increasing complexity, even including situations which may not be frequently encountered in the real oceans.

2.3a NCS: $\lambda=0 ; W=0 ; K$ and $J$ depth independent: The steady state tracer equation:

$$
K_{0} \frac{\partial^{2} C}{\partial z^{2}}+J_{0}=0
$$

or

$$
\frac{\partial^{2} C}{\partial z^{2}}+\frac{J_{0}}{K_{0}}=0
$$

has solutions for $C(z)$ which are functions of the ratio $J_{0} / K_{0}$, for a non-zero $K_{0}$. This ratio along with the boundary conditions completely determines the depth profile, $C(z)$. For $J_{0}=0$, the concentration profile within the mixing zone $\left\{z_{1}<z<z_{2}\right\}$ is a straight line with concentrations $C_{1}$ and $C_{2}$ at the two boundaries. The straight line implies a constant flux at all depths towards the boundary with the lower concentration (also, the flux entering into the mixing zone from below $z_{2}$ equals that leaving from the zone at $z_{1}$ ). All solutions of equations with finite $K_{0}$ and $J_{0}$ will lie on the right of this straight line whether $C_{2}>C_{1}$ or $C_{1}>C_{2}$. The zone on the left is 'forbidden' (see figure 1 ) for the case of constant $K$ and constant or depth dependent $J$ (figure 2).

Let us now discuss in detail the case, $C_{2}>C_{1}$, which holds for nutrients in the oceans. If the $J_{0}$ flux is increased (such that $0<J_{0} / K_{0}<2\left(C_{2}-C_{1}\right) /\left(z_{2}-z_{1}\right)^{2}$ ), the 


\section{Concentration $\left(\mu \mathrm{Mol} / \mathrm{m}^{3}\right.$ )}

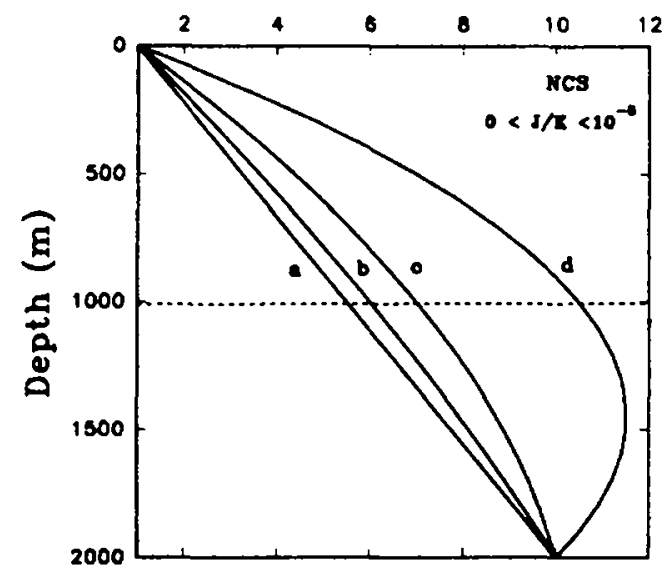

Fizure 1, Depth profiles of a stable non-conservative tracer for different values of $J_{0} / K_{0}$ ( $J$ and $K$ depth independent) in $\mu$ moles $/ \mathrm{m}^{3}$ : (a) 0 (b) $10^{-6}$ (c) $3 \times 10^{-6}$ and (d) $10^{-3}$. The region to the left of $a$ is forbidden. $C_{1}$ and $C_{2}$ are taken as 1 and $10 \mu \mathrm{moles} / \mathrm{m}^{3}$ respectively at depths 1 and $2000 \mathrm{~m}$. Note that the maximum occurs at depths greater than the mid-point of the depth zone, shown by a dotted line.

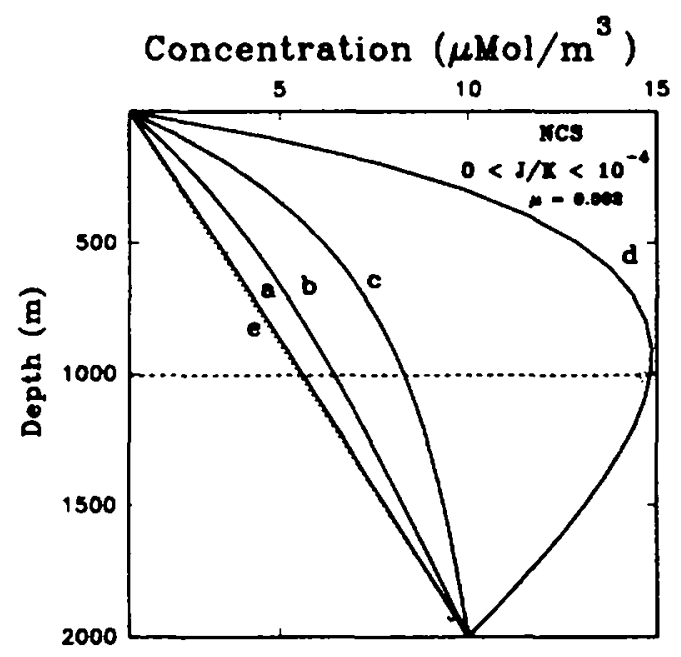

Figure 2 Depth profiles of a stable non-conservative tracer with depth independent $K$ and depth dependent $J$ for different values of $J_{0} / K_{0}\left(\mu\right.$ moles $\left./ \mathrm{m}^{5}\right)$. $J$ varies as $J_{0} \exp (-\mu z)$, with $\mu=0-002 / \mathrm{m}$ (a) $10^{-6}$ (b) $10^{-3}$ (c) $3 \times 10^{-5}$ (d) $10^{-4}$ and (e) 0 . Boundary conditions same as in figure 1 . Note that the maximum occurs at depths less than the mid-point of the depth zone, shown by a dotted line.

concentration gradient and therefore the upward flux at all depths remains the same except in the upper and lower layers of the mixing zone; at the top, the upward flux would increase due to an increase in the concentration gradient (as $C_{1}$ is fixed), and would decrease at the bottom (since $C_{2}$ is fixed). This increased upward flux takes care of the excess $J$ flux in the mixing zone. But if $J_{0}$ flux is increased further (upto 
the limit $\left.J_{0} / K_{0}=2\left(C_{2}-C_{1}\right) /\left(z_{2}-z_{1}\right)^{2}\right)$, the net flux at the bottom (depth $z_{2}$ ) becomes zero and all the tracer introduced by the term $J_{0}$ is removed upwards through $z_{1}$. For values of $J_{0} / K_{0}>2\left(C_{2}-C_{1}\right) /\left(z_{2}-z_{1}\right)^{2}$, there is an upward flux at the top and a downward flux at the bottom (a maximum in concentration develops within the mixing zone at a depth given by $\left(z_{1}+z_{2}\right) / 2+\left(K_{0} / J_{0}\right)\left(C_{2}-C_{1}\right) /\left(z_{2}-z_{1}\right)$. Depending on whether $C_{2}>C_{1}$ or $C_{1}>C_{2}$, the maximum lies either below or above the middepth). This is because the increased addition of tracer at intermediate and greater depths leads to a negative concentration gradient, and consequently a downward flux from the lower end of the mixing zone (depth $z_{2}$ ). As $J_{0}$ is further increased, the negative concentration gradient at depths close to $z_{2}$ extends upwards to shallower and shallower depths, till the integrated $J$ flux in the mixing zone $\left[J_{0}\left(z_{2}-z_{1}\right)\right]$ is removed by equal outward fluxes $\left[J_{0}\left(z_{2}-z_{1}\right) / 2\right]$ at the top and bottom of the mixing zone, a stage at which the depth of reversal in the concentration gradient (or depth of maximum tracer concentration) moves up to the center of the mixing zone, $z=\left(z_{1}+z_{2}\right) / 2$. In figures 1 and 2 , the depths of maximum tracer concentration in the mixing zone lie respectively below and above the mid-depth for the range of parameters considered.

Equation (7) with depth independent $J$ and $K$ parameters does not represent the real ocean, since in the real ocean, $K$ and $J$ display marked depth dependence. When this variation is considered in equation (7), the depth profiles of the nutrients change appreciably. The 'forbidden' zone on the left hand side of the straight line defined by the concentrations at the two boundaries becomes an 'allowed' zone.

2.3b NCS: $\lambda=0 ; W=0 ; K$ and $J$ depth dependent: We will now consider the real ocean model, as we designate it, in which we assume exponential depth dependence of both $K$ and $J$, as supported by experimental data. The steady state equation for this model, for a stable non-conservative tracer (NCS) is:

$$
\frac{\partial}{\partial z}\left\{K \frac{\partial C}{\partial z}\right\}+J=0
$$

For simplicity, the depth dependence of $K$ and $J$ are assumed to be of the form:

$$
K=K_{0}+K_{w} \exp (-\gamma z)
$$

where $\gamma$ is the reciprocal of the scale length for wind induced $K$ variation.

$$
J=J_{0} \exp (-\mu z)
$$

where $\mu$ is the reciprocal of the scale length of the dissolution flux. $J_{0}$ is a constant analogous to $K_{W}$, the values at the top of the zone $(z=0)$ modelled for nutrient/tracer balance.

It can be readily seen that keeping $K$ depth invariant and $J$ depth variable (equation 10), produces similar concentration profiles as in case (a) (figure 2), but the intermediate-depth concentration maximum appears for higher values of $J_{0} / K_{0}$ than in case (a). This is because the addition of the $J$-flux is mostly now in the upper layers of the mixing-zone and a larger increase in $J_{0}$ (or smaller $\mu$ ) is required to produce a reversal in the concentration gradient at intermediate depth which would lead to a downward flux at the bottom. Further, the depth of maximum concentration of 


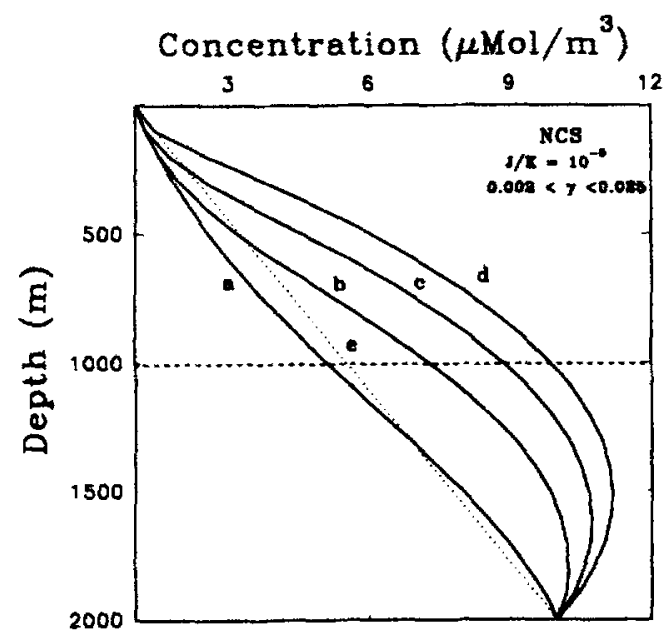

Figure 1. Depth profiles of a stable non-conservative tracer with depth independent $I$ and depth dependent $K\left(\mathrm{~m}^{2} / \mathrm{s}\right)$ for different values of $\gamma\left(\mathrm{m}^{-1}\right)$. $K$ varies as $K_{0}+K_{W} \exp (-\gamma z)$. (a) 0.002 (b) 0.005 (c) 0.01 (d) 0.025 . $K_{0}$ is taken to be $10^{-4} \mathrm{~m}^{2} / \mathrm{s}, K_{w}$ as $9 \times 10^{-4} \mathrm{~m}^{2} / \mathrm{s}$ and $J_{0}$ as $10^{-9} \mu$ moles $/ \mathrm{m}^{3}$.s., except in (e), where it is 0 . Boundary conditions same as in figure 1 . Inclusion of advection $\left(W, 10^{-5}\right.$ to $\left.10^{-7} \mathrm{~m} \mathrm{~s}^{-1}\right)$ does not change the profile significantly.

the tracer now depends on $\mu$ as well, and can occur above mid-depth, even when $C_{2}>C_{1}$. Naturally, the concentration gradient and the tracer flux are greater at the top of the mixing zone than at the bottom.

When $J$ is kept depth invariant and $K$ is varied with depth as in equation (9), the concentration values in the top layers of the mixing zone enter the "forbidden' region (figure 3), due to more efficient removal, and consequent higher fluxes at the upper levels. The effect of this is to reduce the concentration gradient at these levels. It can be seen that by setting $J_{0}=0$, the upward tracer flux is constant throughout the mixing zone. Since the flux is numerically equal to the product of $K$ and the concentration gradient, with $K$ decreasing with depth, the concentration gradient is smaller in the top layers. Lesser the value of $\gamma$, deeper from $z_{1}$ extends the zone of reduced concentration gradient. For $J_{0}>0$, the in situ tracer flux is more efficiently removed at the top layers. At greater depths, the effect of the wind induced $K$ is barely felt (depending on the chosen value of $\gamma$ ) and therefore the concentration maximum is pushed downwards. Obviously, in a steady state situation bulk of the $J$-flux is removed upwards.

Figure 4 shows the concentration profiles of a stable non-conservative tracer, with both $K$ and $J$ varying with depth, for different values of $\gamma$ and $\mu$. The effect of decreasing $\gamma$ is to increase the depth scale of the wind-induced mixing and the concentration gradients near the top of the mixing zone become steeper, for constant values of $\mu, J_{0}, K_{W}$ and $K_{0}$. Similarly, decreasing $\mu$ leads to enhanced addition of tracer at deeper levels for constant values of $\gamma, J_{0}, K_{0}$ and $K_{W}$ and the concentration gradients at the lower end of the mixing zone are reduced. These two situations of decreased $\gamma$ and $\mu$ favour the upward removal of the tracer added by the $J$-flux, into the euphotic zone. 


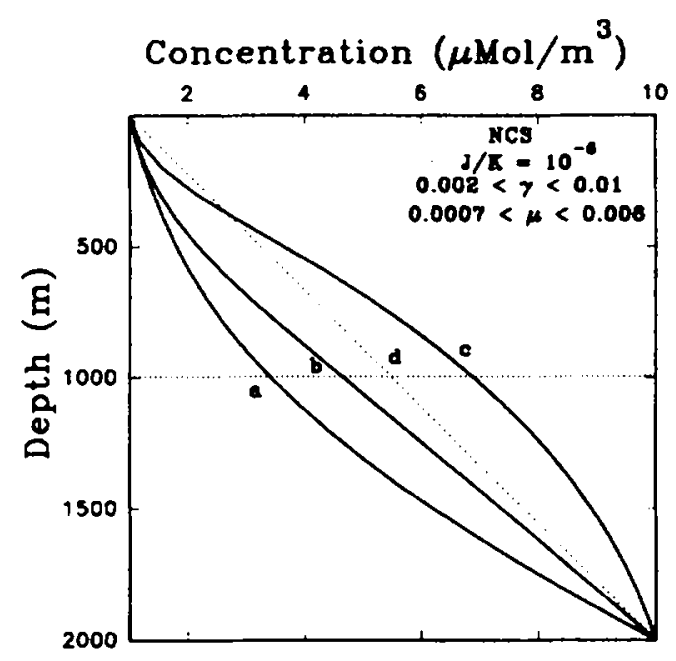

Figure 4. Depth profiles of a stable non-conservative tracer with depth dependent $K$ and $J$ (depth dependence as in figures 2 and 3 ) for different combinations of $\gamma\left(m^{-1}\right)$ and $\mu\left(m^{-1}\right)$ : (a) 0-002,0-006 (b) 0-005, 0-002 and (c) 0-01,0-0007. Other values are as in figure 3 except in

(d) for which $J_{0}=0$ and $\gamma=0$.

2.3c NCR: $\lambda \neq 0 ; W=0 ; K$ and $J$ depth independent. The steady state equation for a non-conservative radiotracer is:

$$
K_{0} \frac{\partial^{2} C^{*}}{\partial z^{2}}-\lambda C^{*}+J_{0}^{*}=0
$$

and its solution is:

$$
C^{*}(z)=c \exp (m z)+d \exp (-m z)+\left(J_{0}^{*} / \lambda\right)
$$

where, $c$ and $d$ are constants determined from boundary conditions and

$$
m=\left(\lambda / K_{0}\right)^{1 / 2}
$$

Assuming no isotopic fractionation during the dissolution, $J_{0}^{*}$ is related to $J_{0}$ by a constant (fractionation factor), which can be assumed to be known from measurements on the organic matter produced in the euphotic zone. Comparing the solutions of equations (11) and (7), we see that $J_{0}$ and $K_{0}$ can be independently estimated using the depth profiles of two non-conservative tracers (a stable and a radioactive isotope of the same element, e.g., ${ }^{14} \mathrm{C}$ ), wherever the model is valid. However, as mentioned before, $K$ and $J$ vary with depth in the real ocean.

2.3d NCR: $\lambda \neq 0 ; W=0 ; J$ and $K$ depth dependent. First let us consider $K$ as depth invariant and $J$ varying with depth as per equation (10). The solution of the equation is:

$$
C^{*}(z)=e \exp (m z)+f \exp (-m z)+\left[J_{0}^{*} /\left(\lambda-\mu^{2} K_{0}\right)\right] \exp (-\mu z)
$$

where $m$ is given by (13). The corresponding solution for a stable tracer is:

$$
C(z)=g+h z-\left(J_{0} / \mu^{2} K_{0}\right) \exp (-\mu z)
$$


The constants $e, f, g$ and $h$ are known from boundary conditions and the fractionation factor $\left(R=J_{0}^{*} / J_{0}\right)$ from surface measurements. For values of $\mu$ such that $\mu \gg m$, we can write:

$$
C^{\star}(z)-R C(z)=e \exp (m z)+f \exp (-m z)-R(g+h z)
$$

This is a function of only $K_{0}$, which can be determined. The depth dependence/ independence of $K$ can also be ascertained from this data, as a depth independent $K$ would yield consistent values throughout the depth range considered. Now, knowing $K_{0}, J_{0}$ and $\mu$ can be determined using the depth profiles of $C^{*}(z)$ and $C(z)$ in the least square sense. The condition $\mu \gg m$ implies that the dissolution scale length $(1 / \mu)$ is much smaller than the mean distance of diffusive transport of one mean life of the tracer, $\left[\sqrt{ }\left(K_{0} \tau\right)\right]$, where $\tau$ is the mean life of the radiotracer. For a long-lived radiotracer such as ${ }^{14} \mathrm{C}$, the mean distance of diffusive transport in one mean life is of the order of $16 \mathrm{~km}$, taking a value of $K_{0}=1 \mathrm{~cm}^{2} / \mathrm{s}$. Therefore the above approximation is valid for ${ }^{14} \mathrm{C}$. This may also hold for shorter-lived tracers, if most of the dissolution occurs in the top few hundred meters, of course depending on the values of $K_{0}$ and half-life of the tracer.

In situations where the above approximation is not valid, the following procedure may be adopted. In equation (15) two parameters $\left(J_{0} / K_{0}\right)$ and $\mu$ can be iteratively determined using the non-conservative stable tracer profile. If these ratios, along with $R$ are substituted in (14), it can be seen that it is only a function of $K_{0}$, which can be estimated from the depth profile of the non-conservative tracer. Thus, $J_{0}, K_{0}$ and $\mu$ can be separately estimated.

In the case of depth dependent $K$, it is difficult to obtain analytical solutions for $C(z)$ and $C^{*}(z)$ and therefore it is difficult to visualize the separation of the constants. However, it is possible to estimate them by least square minimisation in the parameter space.

Figures 5 and 6 show the depth profiles of non-conservative radiotracers $\left({ }^{14} \mathrm{C}\right.$ and ${ }^{32} \mathrm{Si}$ ) for the different cases considered above. For convenience we have drawn a straight line between the two boundary concentration values; this gives the expected behaviour for a stable conservative tracer (curve $e$ ). The region on the left of this line is forbidden for such a tracer, as discussed earlier, for depth independent $K$ values. For a CR tracer, the $-\lambda C^{*}$ term will cause the shift of the profile into the forbidden zone. Likewise, for a NCR tracer, depending on whether $J_{0}^{*}$ is greater or less than $\lambda C^{*}$, the profile will be outside or inside the forbidden zone. This can be seen from curves $a$ in figures 5 and 6 , which show the profiles with $J, K$ independent of depth, with $J_{0}^{*}>0$. When $K$ is depth dependent and $J$, depth independent, the profiles are shown by curves $b$. As $K$ is greater in the top of the mixing zone, the tracer is more efficiently removed and the concentration gradient reduces in the top of the mixing zone. Curves $c$ and $d$ respectively show the profiles for depth independent $K$, depth dependent $J$; and depth dependent $K, J$.

2.3e NCR: $\lambda \neq 0, W \neq 0 ; W, K$ and $J$ depth independent. So far we have not considered the effect of $W$, the advection velocity. A negative $W$ (upwelling) would increase the upward tracer flux, thereby acting in the same direction as the eddy diffusion, if $C_{2}>C_{1}$. However, where a profile shows a maximum at intermediate depths, a negative $W$ will oppose the downward diffusive transport below this 

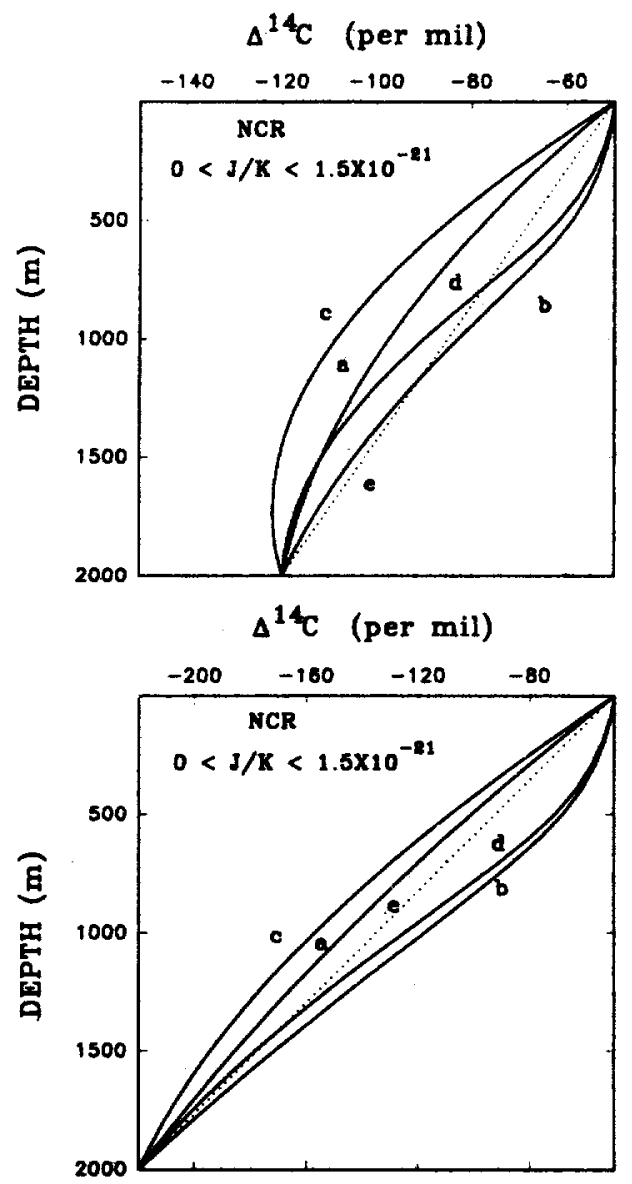

Figare 5. Depth profiles for the non-conservative radiotracer ${ }^{14} \mathrm{C}$. (a) depth independent $K$ and $J^{*}(b)$ depth dependent $K$ and depth independent $J^{*}$ (c) depth independent $K$ and depth dependent $J^{*}$ (d) both $K$ and $J^{*}$ depth dependent. The dotted line (e) betwoen the two boundary concentrations values represents the expected depth profile for a conservative stable tracer. The surface concentrations are set at $-50 \%$ and the deep values at $-220 \%$ and $-120 \%$ to correspond to the actual values observed (figure 7). The value of $J$ was fixed at $1.5 \times 10^{-17} \mathrm{~mol} \mathrm{~m}^{-3} \mathrm{~s}^{-1}$ (sec text).

maximum. The steady state tracer equation for this case is:

$$
K_{0} \frac{\partial^{2} C^{*}}{\partial z^{2}}-W_{0} \frac{\partial C^{*}}{\partial z}-\lambda C^{*}+J_{0}^{*}=0
$$

The solution is:

$$
C^{*}(z)=p \exp (\alpha z)+q \exp (\beta z)+J_{0}^{*} / \lambda
$$

where $\alpha, \beta$ are given by (5). For the stable tracer $(\lambda=0)$ the solution becomes:

$$
C(z)=r \exp \left[\left(W_{0} / K_{0}\right) z\right]+\left(J_{0} / W_{0}\right) z+\left(K_{0} / W_{0}\right)\left[\left(J_{0} / W_{0}\right)-s\right]
$$

where $p, q, r$ and $s$ are constants determined by boundary conditions on $C$ and $C^{*}$. 

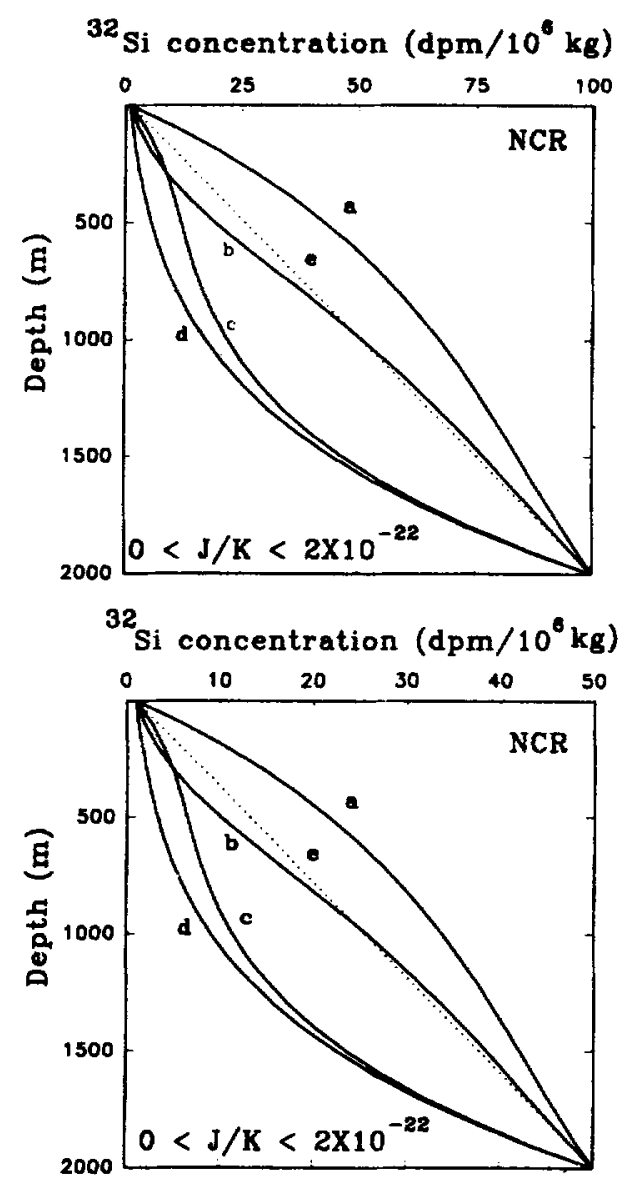

Figure 6. Depth profiles of the non-conservative radiotracer $\left({ }^{3} \mathrm{Si}\right)$. Curves a through $e$ are for the same conditions as in figure 5 . The surface concentrations are set at $1 \mathrm{dpm} / 10^{6} \mathrm{~kg}$ and the deep values at 50 and $100 \mathrm{dpm} / 10^{6} \mathrm{~kg}$ to correspond to the actual values observed (figure 8). The value of $J$ was fixed at $1.5 \times 10^{-23} \mathrm{~mol} \mathrm{~m}^{-3} \mathrm{~s}^{-1}$.

Now if we determine $\left(W_{0} / K_{0}\right)$ using a stable conservative tracer (SC) as discussed in (2.1), using (19) (NCS) we can determine $\left(J_{0} / W_{0}\right)$; knowing $R$, the fractionation factor, and substituting the above ratios in (18), we see that the profile of NCR (18) can be used to estimate $W_{0}$. Thus, $K_{0}, J_{0}$ and $W_{0}$ can be separately estimated using a combination of conservative stable, non-conservative stable and non-conservative radiotracers.

If $J$ and $K$ are depth dependent, then it is difficult to obtain analytical solutions to the equations. In such cases, the physical parameters of interest may be estimated using a suite of tracers by least square minimisation.

\section{Tracer data}

Recently four new radiotracers, ${ }^{32} \mathrm{P},{ }^{33} \mathrm{P},{ }^{7} \mathrm{Be}$ and ${ }^{234} \mathrm{Th}$ have been used to study the nutrient cycling in the upper ocean (Coale and Bruland 1985; Lal and Lee 1988; 
Lal et al 1988; Waser 1993). The concentrations of these radiotracers have been measured in plankton as well as in the water column in the Southern California Bight (SCB) using newly developed techniques. Amongst these, only ${ }^{7} \mathrm{Be}$ behaves as a conservative tracer (refer Lee et al 1991) and primarily is an indicator of diffusivity in the water column (Silker 1972; Young and Silker 1974). From depth profiles of ${ }^{7} \mathrm{Be}$, the mean eddy diffusivity in the SCB waters was estimated to be $\sim 7 \mathrm{~cm}^{2} / \mathrm{s}$ from two profiles. This, when coupled with the nitrate profile in the water column can be used to estimate the new production, $\sim 1 \mathrm{~g} \mathrm{C} \mathrm{m}^{-2} \mathrm{day}^{-1}$ (Lee et al 1991). Simultaneous studies of cosmogenic ${ }^{32} \mathrm{P}$ and ${ }^{33} \mathrm{P}$ and radiotracers ${ }^{234} \mathrm{Th}$ along with ${ }^{7} \mathrm{Be}$ can provide detailed insight about the various parameters entering the mixing equations considered. The incorporation of $\mathrm{P}$ in the sinking organic particles appreciably depletes the inventories of ${ }^{32} \mathrm{P}$ and ${ }^{33} \mathrm{P}$ in the surface waters, over and above that due to mixing of water by eddy diffusivity.

Several radiocarbon profiles were obtained during the GEOSECS and INDIGO expeditions. In figure 7 we show the $\Delta^{14} \mathrm{C}$ profiles in the Indian, Atlantic and Pacific Oceans (Ostlund and Stuiver 1980; Stuiver and Ostlund 1980; Bard et al 1988). The near surface values have been corrected for the addition of bomb ${ }^{14} \mathrm{C}$, fixing the pre-bomb surface value at $-50 \%$. We may now compare these profiles with those in figure 5 , where we have already chosen the two boundary conditions to match

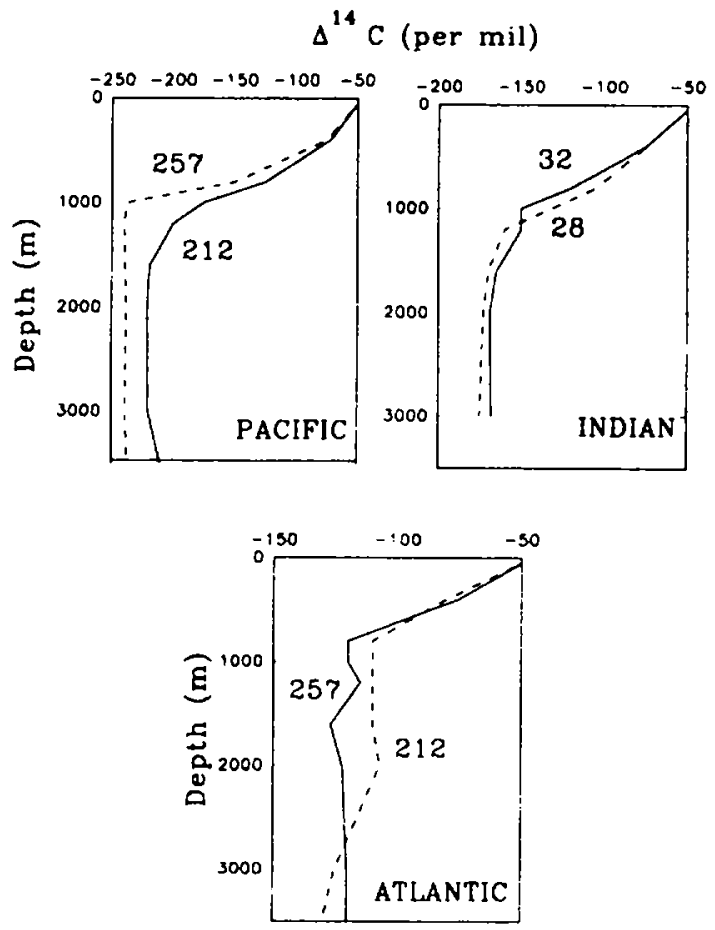

Figure 7. Depth profiles of $\Delta^{14} \mathrm{C}$ in the major world oceans. Surface values have been corrected for the addition of bomb ${ }^{14} \mathrm{C}$. The Indian Ocean pre-bomb values are from Bard et al 1988; The Pacific data from Ostlund and Stuiver (1980) and the Atlantic data from Stuiver and Ostlund (1980). Station locations are: $28\left(17^{\circ} 20^{\circ} \mathrm{N}, 54^{\circ} 50^{\circ} \mathrm{E}\right) ; 32\left(12^{\circ} 20^{\circ} \mathrm{S}\right.$, $\left.53^{\circ} 40^{\circ} \mathrm{E}\right) ; 107\left(12^{\circ} \mathrm{S}, 2^{\circ} \mathrm{E}\right) ; 49\left(7^{\circ} 56^{\prime} \mathrm{S}, 28^{\circ} 12^{\prime} \mathrm{E}\right) ; 212\left(30^{\circ} \mathrm{N}, 159^{\circ} 50 \mathrm{~W}\right)$ and $257\left(10^{\circ} 10 \mathrm{~S}\right.$, $\left.169^{\circ} 58^{\prime} \mathbf{W}\right)$. 

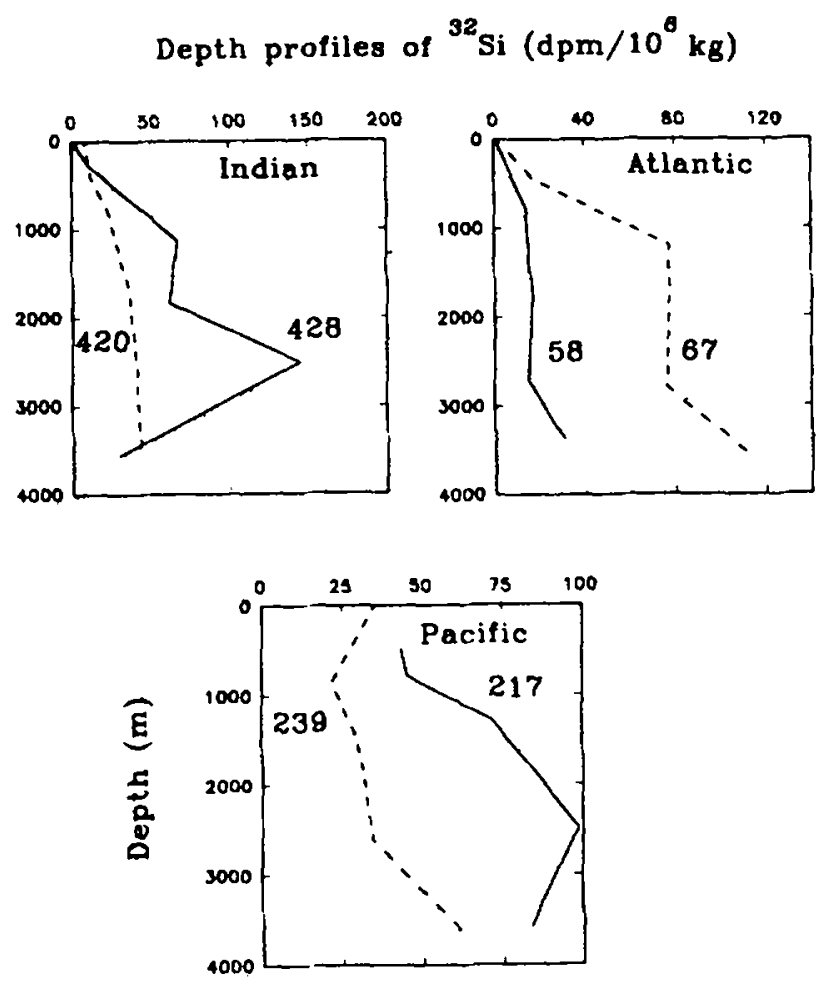

Figure 8. Depth proliles of ${ }^{32} \mathrm{Si}\left(\mathrm{dpm} / 10^{6} \mathrm{~kg}\right)$ in the major world oceans. The data from

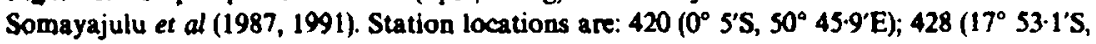
$\left.57^{\circ} 41^{\prime} \mathrm{E}\right) ; 239\left(5^{\circ} 59 \cdot 1^{\prime} \mathrm{N}, 172^{\circ} \mathrm{W}\right) ; 217\left(44^{\circ} 36 \cdot 7^{\prime} \mathrm{N}, 176^{\circ} 49.9^{\prime} \mathrm{W}\right) ; 58\left(27^{\circ} \mathrm{S}, 37^{\circ} 1.4^{\prime} \mathrm{W}\right)$ and $67\left(44^{\circ} 58^{\prime} \mathrm{S}, 51^{\circ} 3 \cdot 5^{\prime} \mathrm{W}\right)$.

the experimental values. One sees a fairly good overall agreement with the theoretical curve ' $d$ ', implying that both the eddy diffusivity and the dissolution flux are strongly depth dependent in the upper $1 \mathrm{~km}$. The concentration gradients are small at depths $>1 \mathrm{~km}$. Station 28 in the Indian Ocean does not show any significant effect of the Antarctic intermediate waters, justifying the application of the 1-D model in this case.

Figure 8 shows the GEOSECS ${ }^{32} \mathrm{Si}$ profiles in the major world oceans (Somayajulu et al 1987 and 1991). Due to the necessity of large volume filtration for ${ }^{32} \mathrm{Si}$ measurements, a few data points are availablo in each profile and the depth resolution is therefore very coarse. Nevertheless, the broad similarities with our theoretical profiles can be readily seen (figure 6). Different cases in the observed profiles are represented in the theoretical curves using 1-D model. Somayajulu et al (1991) estimated the eddy diffusivity for Indian Ocean stations 420 and 428 and Pacific Ocean station 239 (seo figure 8 ) to be $\sim 16,2$ and $3 \mathrm{~cm}^{2} \mathrm{~s}^{-1}$ respectively. The $K$ values used in calculations (figure 6), envelop these estimates.

Figure 9 shows the depth profiles of non-conservative stable tracers $\mathrm{PO}_{4}, \mathrm{NO}_{3}$ and $\mathrm{SiO}_{2}$ in the SCB region (CALCOFI data), one of the most well studied regions of the world (Eppley 1986). These profiles resemble the theoretical profiles shown in figure 2. 


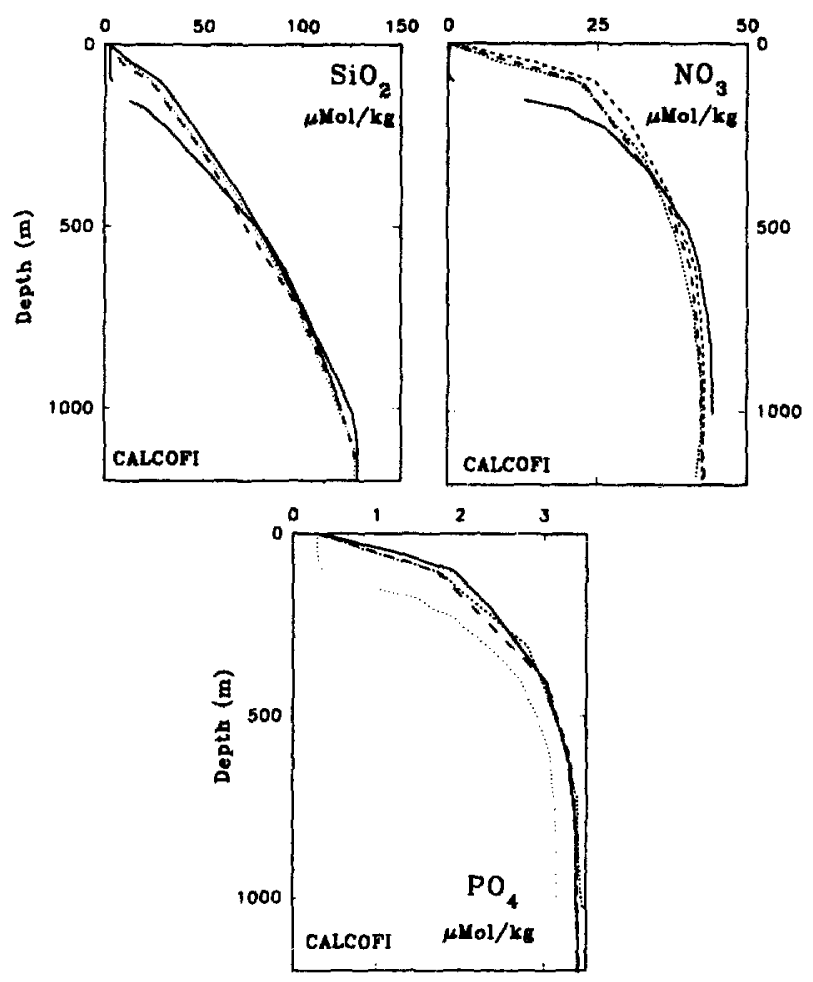

Figure 9. Depth profiles of phosphate, silicate and nitrate in the Southern California Bight (CALCOFI data) for a 2 year period, 1983-1985, covering winter and summer seasons. These profiles closely resemble the theoretical profiles shown in figure 2.

\section{Conclusions}

We have theoretically considered the problem of interpretation of the role of nonconservative tracers for biological processes in the upper ocean. These tracers by definition include new additional parameters circumscribed by their chemical behavior in the ocean, but of course when tracers which belong to the nutrient elements (e.g., $\mathrm{N}, \mathrm{P}, \mathrm{Si}$ ) are used, the new parameters are the ones sought after to understand the nutrient cycling. Besides estimation of these parameters, the interpretation of the tracer data involves explicit knowledge of the mixing processes and their rates, which in our treatment have been configured by the parameters $K$ and $W$.

The circulation and mixing in the upper layers of the ocean is governed principally by the eddy diffusion parameter, $K$. The parameter $W$ becomes important when dealing with large space and time scales, comparable to the circulation time of the oceans. The stable isotopes of hydrogen and oxygen can be used for characterizing principal water masses, but they are not useful for determining the eddy diffusivity, specifically in the upper ocean. The natural tracer, ${ }^{3} \mathrm{H}$ (half life $\sim 12$ years), is in principle useful for this purpose but its extremely low concentration prohibits a meaningful application.

The useful principal radiotracers, in the order of decreasing half lives are: ${ }^{14} \mathrm{C}$, ${ }^{226} \mathrm{Ra},{ }^{32} \mathrm{Si},{ }^{7} \mathrm{Be},{ }^{210} \mathrm{~Pb},{ }^{234} \mathrm{Th},{ }^{32} \mathrm{P}$ and ${ }^{222} \mathrm{Rn}$. Except for $\mathrm{N}$, other nutrient elements 
(C, Si, P) have natural radiotracers, which can be used along with other stable and radiotracers to characterize the nutrient cycles. The tracer $\mathrm{Ra}$ imitates chemically the behavior of $\mathrm{Ba}$, which is enriched in plankton. Tracers like Th and Po are quickly absorbed on to particulate organic matter and may, in some cases, provide limits on the new production (Chung and Craig 1973; Sarin et al 1994).

Our discussions, which include comparisons of model profiles with those obtained for different tracers, show that it is possible to obtain quantitative estimates of the dissolution flux and eddy diffusivities in the upper ocean by a suitable combination of non-conservative stable and radiotracer data. The importance of these studies can hardly be overemphasized. We believe that interrelationships between nutrient cycling processes and their temporal variability are essential for understanding the temporal variability in new production, total productivity, etc. Long time series of sediment trap data in different oceanic regions are not useful unless basic processes are understood.

\section{References}

Bard E, Arnold M, Ostlund H G, Maurice P, Monfray P and Duplessy J C 1988 Penetration of bomb radiocarbon in the tropical Indian Ocean measured by means of accelerator mass spectrometry; Earth Planet. Sci. Lett. 87 379-389

Coale K H and Bruland K W $1985^{234} \mathrm{Th}:{ }^{238} \mathrm{U}$ disequilibria within the California current; Limn. Oceanogr. 30 22-23

Broecker W S and Peng T H 1982 Tracers in the Sea, (Palieades, NY: Eldigio Press) pp. 690

Craig H 1969 Abyssal carbon and radiocarbon in the Pacific; J. Geophys. Res. 745491

Chung Y and Craig H $1973{ }^{226} \mathrm{Ra}$ in the eastern equatorial Pacific; Earth Planet. Sci. Lett. 17 306-318

Eppley R W 1986 Plankton dynamics of the southern California Bight (Berlin: Springer-Verlag)

Lal D 1962 Cosmic ray produced radionuclides in the sea; J. Oceanogr. Soc. Jpn. 20 600-604

Lal $D$ and Lee $T 1988$ Cosmogenic ${ }^{32} \mathrm{P}$ and ${ }^{33} \mathrm{P}$ used as tracers to study phosphorus recycling in the upper ocean; Nature 333 752-753

Lal D, Chung Y, Platt T and Lee T 1988 Twin cosmogenic radiotracer studies of phosphorus recycling and chemical fluxes in the upper ocean; Limn. Oceanogr. 33 1559-1567

Lee T, Barg E and Lal D 1991 Techniques for extraction of dissolved inorganic and organic phosphorus from large volumes of sea water, Anal. Chim. Acta. 260 113-121

Munk W H 1966 Abyssal recipes; Deep-Sea Res. 13 707-730

Ostlund H G and Stulver M 1980 GEOSECS Pacific Radiocarbon; Radiocarbon 22 1-24

Ramesh R and Lal D 1994 Analysis of non-steady state nutrient profiles; paper in preparation for JGOFS special volume of Proc. Indian Acad. Sci. (Earth Planet Sci.)

Sarin M M, Krishnaswami S, Ramesh R and Somayajulu B L K $1994{ }^{238}$ U series nuclides in the northeastern Arabian Sea: Scavenglng rates and cycling processes; Continental Shelf Research (in press)

Silker W B 1972 Horizontal and vertical distributions of radionuclides in the North Pacific Ocean; $J$. Geophys. Res. 77 1061-1070

Somayajulu B L K, Rengarajan R. Lal D, Weiss R F and Craig H 1987 GEOSECS Atlantic Si-32 profiles; Earth Planet. Sci. Lett. 85 329-342

Somayajulu B L K, Rengarajan R, Lal D and Craig H 1991 GEOSECS Pacific and Indian Si-32 profiles; Earth Planet. Sci. Lett. 107 197-216

Stuiver M and Ostlund H G 1980 GEOSECS Atlantic Radiocarbon; Radiocarbon 22 25-53

Waser N A D 1993 Cosmogenic ${ }^{32} \mathrm{P}$ and ${ }^{33} \mathrm{P}$ in the atmosphere and oligotrophic ocean and applications to the study of phosphorus cycling (Woods Hole: MIT) Unpublished Ph. D. thesis

Young $J A$ and Silker W B 1974 The determination of air-sea exchange and oceanic mixing rates using 'Be during the Bormex experiment; J. Geophys. Res. $794481-4489$ 